\title{
Arthroscopic Treatment of Iliotibial Band Syndrome
}

\author{
Courtney H. Cowden III, M.D., and F. Alan Barber, M.D.
}

\begin{abstract}
Lateral knee pain in athletes is commonly seen in the sports medicine clinic, and the diagnosis of iliotibial band (ITB) syndrome is frequently made. Although conservative management including rest from activity, equipment modification, oral nonsteroidal anti-inflammatory drug use, and physical therapy is the mainstay of treatment initially, refractory cases do exist. Multiple surgical techniques have been described including an arthroscopic technique. Arthroscopic release of the ITB attachment to the lateral femoral epicondyle and resection of the lateral synovial recess for recalcitrant ITB syndrome comprise a valid option that can have a good outcome. This option avoids the complications associated with open surgery and allows for a complete arthroscopic knee examination. Division or lengthening of the ITB band itself is not a necessary step in this technique.
\end{abstract}

$\mathbf{R}$ enne $^{1}$ first described iliotibial band (ITB) syndrome in 1975 in the US Marine Corps recruit population. Since that time, the syndrome has been identified in multiple endurance athletes including runners ( $1.6 \%$ to $12 \%$ incidence) and cyclists (15\% to $24 \%$ incidence), as well as multiple other sports. The most common complaint is lateral knee pain, ${ }^{2}$ and patients with ITB syndrome have been shown to have different running kinematics than those without the problem. ${ }^{3}$

Anatomically, the ITB, or iliotibial tract, constitutes a lateral thickening of the fascia lata of the thigh. It is formed from a coalescence of fascial elements from the tensor fascia lata as well as the gluteus maximus and minimus at the level of the greater trochanter. Moving distally, the ITB has attachments to the intermuscular septum and supracondylar tubercle. It then passes over the lateral femoral condyle, crossing the knee joint and inserting on Gerdy's tubercle at the anterolateral proximal tibia. Proximal to the lateral femoral condyle, a layer of fat separates the ITB from underlying structures, but at the condyle, the band is more intimately involved with underlying layers of tissue. ${ }^{2}$

From Plano Orthopedic Sports Medicine and Spine Center, Plano, Texas, U.S.A.

The authors report the following potential conflict of interest or source of funding: F.A.B. receives support from DePuy-Mitek, Linvatec, and Arthrex.

Received June 12, 2013; accepted August 15, 2013.

Address correspondence to F. Alan Barber, M.D., Plano Orthopedic Sports Medicine and Spine Center, 5228 W Plano Pkwy, Plano, TX 75093.

U.S.A.

(C) 2014 by the Arthroscopy Association of North America

$2212-6287 / 13389 / \$ 36.00$

http://dx.doi.org/10.1016/j.eats.2013.08.015
Although conservative management including rest from activity, equipment modification, oral nonsteroidal antiinflammatory drug (NSAID) use, and physical therapy is the mainstay of treatment initially, refractory cases do exist. $^{2}$ Surgical options include percutaneous release, ${ }^{4}$ open release, ${ }^{5,6}$ ITB Z-lengthening, ${ }^{7,8}$ and an arthroscopic technique.

An arthroscopic technique is desirable for many reasons including the inherent capacity to examine and address any associated intra-articular pathology during the same session. It is also inherently less invasive than the open options. Assuming the inflammation in the tissue connecting the ITB band to the lateral femoral epicondyle is the culprit of ITB pain, this tissue can be removed with an arthroscopic shaver. ${ }^{9}$ Michels et al. ${ }^{9}$ described such a technique in a series of 36 patients successfully treated for ITB syndrome and followed up for at least 18 months. This case report illustrates our use of this arthroscopic approach.

\section{Clinical Presentation}

A 41-year-old white man described chronic lateral left knee pain for at least 10 years. He was a marathon runner and ran the Boston Marathon in the previous 5 years. Despite several nonoperative therapies, his pain continued to worsen. In the previous 6 months, these nonoperative therapies included physical therapy, NSAIDs, a steroid injection, and shoe insoles without improvement. Magnetic resonance imaging evaluation obtained immediately before his visit was negative for any significant pathology. He continued to run 15 miles per week with a consistent and appropriate stretching regimen. He reported no other medical problems and did not smoke. He had no complaints of instability. 
Physical examination showed tenderness to pressure over the ITB and the lateral femoral condyle and a positive Ober test. He was diagnosed with ITB syndrome and given a specific home stretching program, additional physical therapy, and $7.5 \mathrm{mg}$ of meloxicam daily. Surgical options were discussed but not recommended.

The patient returned to the clinic 9 months later with no improvement in his symptoms. He requested a surgical option to treat his ITB pain. The treatment plan chosen was an examination under anesthesia followed by an arthroscopic evaluation and debridement of the lateral synovial recess and the ITB band.

\section{Surgical Technique}

Other than a routine arthroscopic setup, no special equipment is necessary to perform this procedure. Although our patient was positioned with the operative leg in a leg holder and hanging off the end of the bed, this procedure could also be performed with the patient fully supported by the bed and using a lateral post. The arthroscopic shaver should be of appropriate size for the volume of tissue to be removed; a ConMed Linvatec 4.2-mm shaver blade (Largo, FL) was used during this case.

The examination under anesthesia did not show any abnormalities. By use of a standard 4-portal technique (central trans-patellar tendon viewing portal, anterolateral instrumentation portal, superomedial secondary fluid inflow portal, and suprasuperior lateral instrumentation portal), the knee joint was completely examined arthroscopically. This standard examination included the medial and lateral compartments, including the posterior medial and posterior lateral recesses, evaluated through the intracondylar notch. No meniscus pathology was observed. The femoral and tibial articular cartilage was without visible defect, and there were no loose bodies.

The arthroscope was then placed in the suprasuperior lateral portal, and the patellofemoral joint was completely evaluated (Fig 1). No patella or trochlea pathology was found. The lateral gutter and synovial recess were also completely visualized (Fig 2). The anterolateral portal continued to be used for instrumentation. The lateral synovial fold was identified and resected with the shaver (Fig 3). This resection (Video 1) was extended to include the area of the lateral epicondyle while also removing soft tissue laterally to reveal the underside of the ITB. The resection continued until the ITB was completely released from the lateral epicondyle (Fig 4). A percutaneously placed needle was used as a probe to confirm the appropriate anatomic location and complete resection of the tissue. Bone was not exposed in an effort to reduce postoperative bleeding.

The portals were closed with No. 3-0 absorbable suture after the intra-articular injection with $0.25 \%$ bupivacaine solution. The knee was wrapped in a soft dressing,

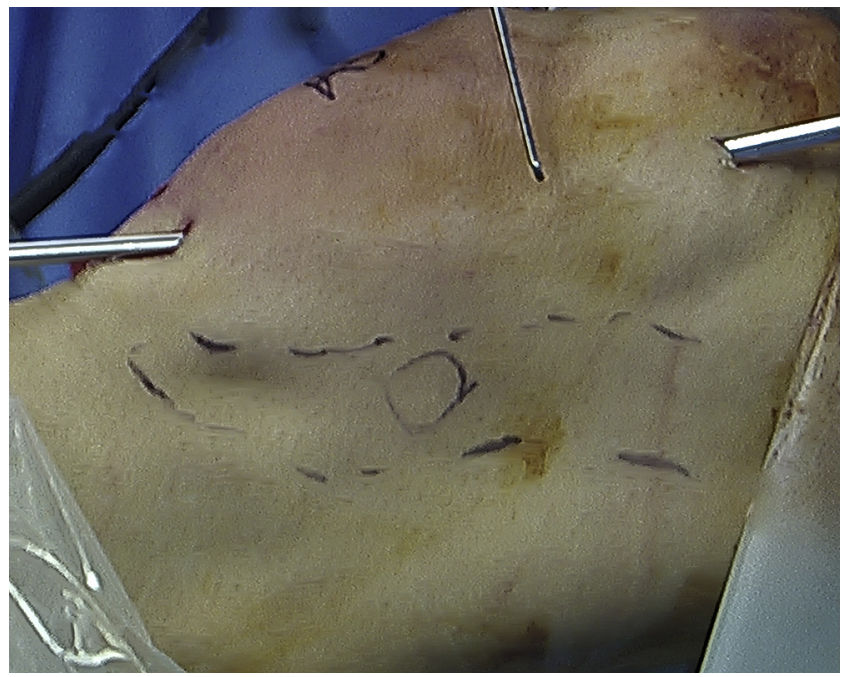

Fig 1. The lateral view of a left knee shows the arthroscope in the suprasuperior lateral portal and the shaver in the inferior lateral portal. The ITB is outlined in dashes, and the circle is the location of the lateral femoral epicondyle.

and the patient was awakened from anesthesia without complication. He was discharged on the same day as surgery and was allowed full activity as tolerated postoperatively. His preoperative pain was completely resolved 4 weeks after surgery, and he returned to his regular athletic activities without difficulty. He had no postoperative complications and no further complaints. He stated that he was satisfied with his result.

\section{Discussion}

Early explanations for the etiology of ITB syndrome included a friction syndrome due to cyclic anterior-

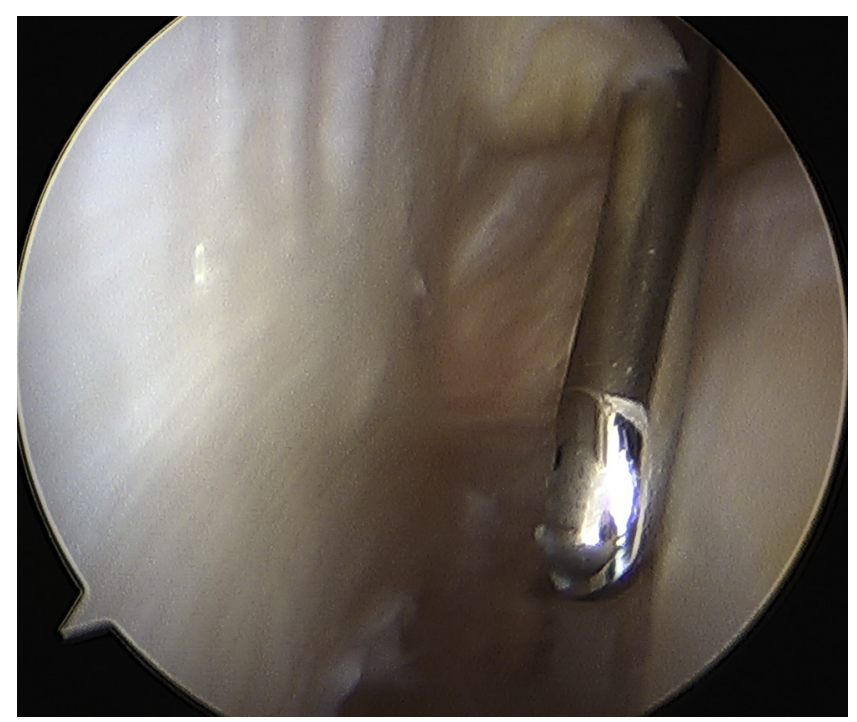

Fig 2. The lateral gutter is visualized from the proximal suprasuperior lateral portal with the shaver in the inferior lateral portal. 


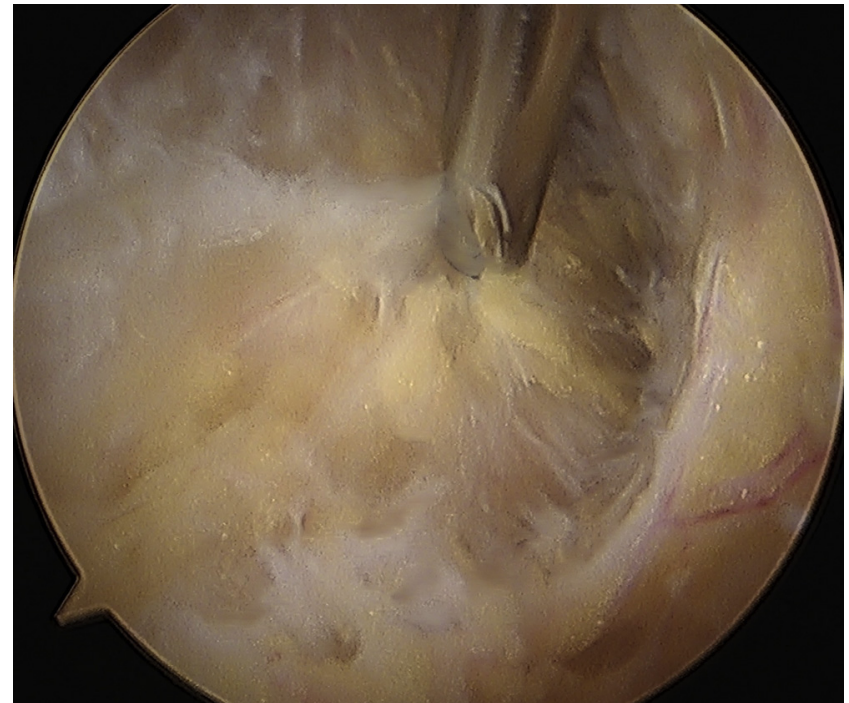

Fig 3. The lateral synovial fold is identified and resected.

posterior motion of the ITB over the lateral femoral epicondyle leading to inflammation and pain., ${ }^{4,10,11}$ This friction-based understanding was supplemented with the concept of the ITB impingement zone, occurring at around $30^{\circ}$ of knee flexion, which was also the most common knee position during foot strike. ${ }^{11}$ Fairclough et al. ${ }^{12}$ refuted this friction model and proposed an etiology based on fascia lata compression through cyclic tension changes within the ITB during gait.

Regardless of loading, other authors have proposed that the pain in ITB syndrome originates in an area of fluid collection located deep to the thickened ITB in the region of the femoral epicondyle noted to be present on magnetic resonance imaging scanning ${ }^{13,14}$ and consistent with a potential space (a bursa) found on cadaveric

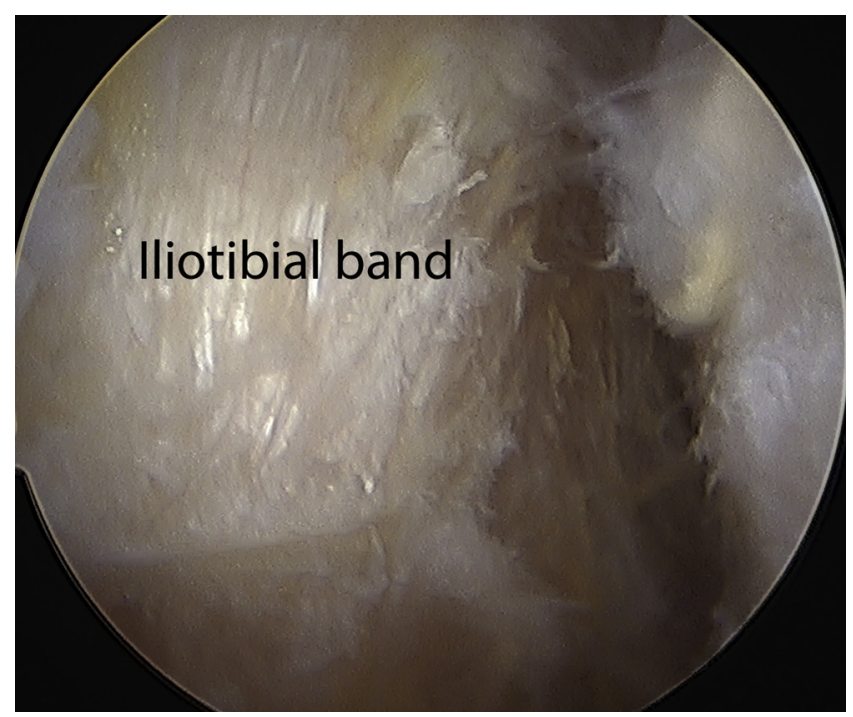

Fig 4. The resection of the ITB attachment to the lateral femoral epicondyle reveals the underside of the ITB. This area can be localized from the outside using a needle probe.
Table 1. Pearls and Pitfalls for Arthroscopic ITB Release

- Viewing the lateral synovial recess from a suprasuperior lateral portal provides a direct viewing advantage over an anteriorinferior lateral portal.

- Use an outside-in spinal needle to confirm the location of the lateral epicondyle.

- Be alert for loose bodies that may hide in the inferior portions of the lateral synovial recess near the popliteus tendon.

- A tourniquet should not be used to avoid impairing postoperative rehabilitation.

- Be mindful that excessive bony abrasion on the lateral condyle may create bleeding, which can impair one's view and increase postoperative swelling and pain.

dissections between the ITB and the knee capsule. ${ }^{13}$ Surgically, others described this same structure as synovium in a "lateral synovial recess," which is actually a lateral extension and invagination of the actual knee joint capsule and not a separate bursa because it is connected to the rest of the knee joint capsule. ${ }^{15}$ Nemeth and Sanders ${ }^{15}$ reported that the inflamed tissue in ITB syndrome is found in and around this lateral synovial recess connecting the lateral femoral condyle to the ITB.

Patients with ITB syndrome will typically present with pain in the region of the distal ITB at various areas, ranging from the lateral femoral condyle to the insertion at the Gerdy tubercle. Initially, the pain will usually be aggravated by activity, but it may progress to pain at rest. ${ }^{2}$ A complete physical examination of the knee should include the Ober test ${ }^{4}$ and Thomas test. ${ }^{16}$ Most surgeons will not attempt a surgical intervention for ITB syndrome until nonoperative therapies have failed for at least 6 months. ${ }^{2}$

Nonoperative management should remain the principal focus for the treatment of ITB syndrome, and most patients with ITB syndrome will show improvement. Our patient was initially treated with a variety of nonoperative modalities including steroid injections, NSAIDs, physical therapy, home stretching programs, and activity modifications before finally being considered a candidate for surgical intervention. ${ }^{2}$ Multiple surgical techniques have been described, ${ }^{4-8}$ including an arthroscopic technique. ${ }^{9}$ The procedure outlined in this report and in the associated video is appropriate for patients who have chronic, recalcitrant complaints and offers an arthroscopic approach that had a good outcome.

Addressing the ITB arthroscopically offers several advantages over other described procedures including open release. The entire joint can be examined in detail, and any other pathology may be concurrently addressed without additional incisions. The lateral synovial recess may be simply debrided, or a percutaneous Z-plasty or release of the ITB may be performed under direct visualization from within the joint. Several pearls are listed in Table 1. 
Our patient's results are consistent with those reported by Michels et al., ${ }^{9}$ who described a similar treatment in 34 knees. In addition, the described procedure is consistent with the evolving and current understanding of the pathogenesis of ITB syndrome. Nemeth and Sanders ${ }^{15}$ confirmed that fibrous and chronically inflamed tissue inhabits the lateral synovial recess in patients with chronic ITB syndrome. Removal of this tissue should address the underlying cause of pain. The ITB is also preserved using this technique. This case presentation is limited by its lack of long-term follow-up and the lack of the use of a validated functional scoring system to assess improvement.

Arthroscopic release of the ITB attachment to the lateral femoral epicondyle and resection of the lateral synovial recess for recalcitrant ITB syndrome comprise a valid option that can have a good outcome. This option avoids the complications associated with open surgery and allows for a complete arthroscopic knee examination. Division or lengthening of the ITB band itself is not a necessary step in this technique.

\section{References}

1. Renne JW. The iliotibial band friction syndrome. J Bone Joint Surg Am 1975;57:1110-1111.

2. Strauss EJ, Kim S, Calcei JG, Park D. Iliotibial band syndrome: Evaluation and management. J Am Acad Orthop Surg 2011;19:728-736.

3. van der Worp MP, van der Horst N, de Wijer A, Backx FJ, Nijhuis-van der Sanden MW. Iliotibial band syndrome in runners: A systematic review. Sports Med 2012;42: 969-992.
4. Holmes JC, Pruitt AL, Whalen NJ. Iliotibial band syndrome in cyclists. Am J Sports Med 1993;21:419-424.

5. Noble CA. Iliotibial band friction syndrome in runners. Am J Sports Med 1980;8:232-234.

6. Martens M, Libbrecht P, Burssens A. Surgical treatment of the iliotibial band friction syndrome. Am J Sports Med 1989;17:651-654.

7. Richards DP, Barber FA, Troop RL. Iliotibial band Zlengthening. Arthroscopy 2003;19:326-329.

8. Barber FA, Boothby MH, Troop RL. Z-plasty lengthening for iliotibial band friction syndrome. J Knee Surg 2007;20: 281-284.

9. Michels F, Jambou S, Allard M, et al. An arthroscopic technique to treat the iliotibial band syndrome. Knee Surg Sports Traumatol Arthrosc 2009;17:233-236.

10. Lavine R. Iliotibial band friction syndrome. Curr Rev Musculoskelet Med 2010;3:18-22.

11. Orchard JW, Fricker PA, Abud AT, Mason BR. Biomechanics of iliotibial band friction syndrome in runners. Am J Sports Med 1996;24:375-379.

12. Fairclough J, Hayashi K, Toumi H, et al. The functional anatomy of the iliotibial band during flexion and extension of the knee: Implications for understanding iliotibial band syndrome. J Anat 2006;208:309-316.

13. Ekman EF, Pope T, Martin DF, Curl WW. Magnetic resonance imaging of iliotibial band syndrome. Am J Sports Med 1994;22:851-854.

14. Murphy BJ, Hechtman KS, Uribe JW, et al. Iliotibial band friction syndrome: MR imaging findings. Radiology 1992;185:569-571.

15. Nemeth WC, Sanders BL. The lateral synovial recess of the knee: Anatomy and role in chronic Iliotibial band friction syndrome. Arthroscopy 1996;12:574-580.

16. Fredericson M, Weir A. Practical management of iliotibial band friction syndrome in runners. Clin J Sport Med 2006;16:261-268. 